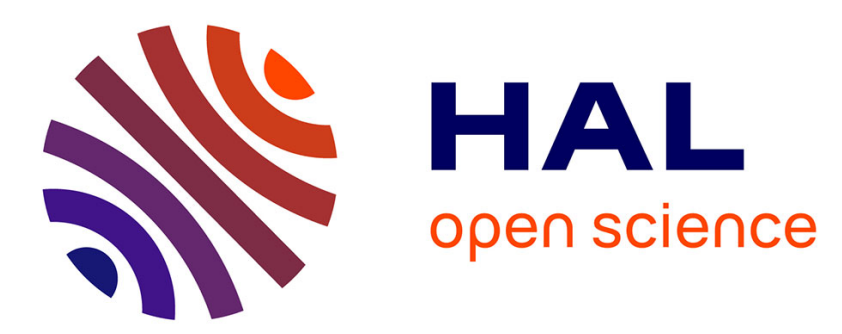

\title{
Validation of full-f global gyrokinetic modeling results against the FT-2 tokamak Doppler reflectometry data using synthetic diagnostics
}

O L Krutkin, A B Altukhov, A D Gurchenko, E. Gusakov, M A Irzak, L A Esipov, A. Sidorov, L. Chone, T. Kiviniemi, S. Leerink, et al.

\section{To cite this version:}

O L Krutkin, A B Altukhov, A D Gurchenko, E. Gusakov, M A Irzak, et al.. Validation of full-f global gyrokinetic modeling results against the FT-2 tokamak Doppler reflectometry data using synthetic diagnostics. Nuclear Fusion, 2019, 59, pp.096017. 10.1088/1741-4326/ab1cfb . hal-02434476

\section{HAL Id: hal-02434476 \\ https://hal.univ-lorraine.fr/hal-02434476}

Submitted on 10 Jan 2020

HAL is a multi-disciplinary open access archive for the deposit and dissemination of scientific research documents, whether they are published or not. The documents may come from teaching and research institutions in France or abroad, or from public or private research centers.
L'archive ouverte pluridisciplinaire HAL, est destinée au dépôt et à la diffusion de documents scientifiques de niveau recherche, publiés ou non, émanant des établissements d'enseignement et de recherche français ou étrangers, des laboratoires publics ou privés. 


\title{
Validation of full-f global gyrokinetic modeling results against the FT-2 tokamak Doppler reflectometry data using synthetic diagnostics
}

\author{
O.L. Krutkin ${ }^{1,4}$, A.B. Altukhov 11 , A.D. Gurchenko 1 , E.Z. Gusakov ${ }^{1}$, \\ M.A. Irzak ${ }^{1}$, L.A. Esipov ${ }^{1}$, A.V. Sidorov ${ }^{1}$, L. Chôné2 ${ }^{2}$ T.P. Kiviniemi ${ }^{2}$,

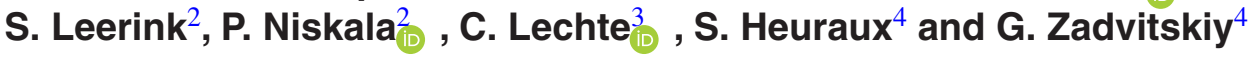 \\ ${ }^{1}$ Ioffe Institute, St Petersburg, Russian Federation \\ 2 Aalto University, Espoo, Finland \\ 3 Institute of Interfacial Process Eng. and Plasma Technology, 70569 Stuttgart, Germany \\ ${ }^{4}$ Institute Jean Lamour UMR 7198 CNRS, Université de Lorraine, BP 50840, 54011 Nancy, France \\ E-mail: a.altuhov@mail.ioffe.ru
}

Published 23 July 2019

\begin{abstract}
Two versions of the X-mode Doppler reflectometry (DR) synthetic diagnostics are developed within the framework of the ELMFIRE global gyrokinetic modeling of the FT-2 tokamak ohmic discharge. In the 'fast' version the DR signal is computed in the linear theory approximation using the reciprocity theorem, utilizing the probing wave field pattern provided by computation and taking into account the 2D plasma inhomogeneity effects; whereas the alternative 'slow' version DR synthetic diagnostic is based on the full-wave code IPF-FD3D describing the probing and scattered wave propagation in turbulent plasma. The DR signal frequency spectra and the dependence of their frequency shift, width and shape on the probing antenna position are computed and shown to be similar to those measured in the high-field side probing DR experiment at the FT-2 tokamak. The geodesic acoustic mode characteristics provided by the measurements and by the synthetic DR are close within a 12\% accuracy. However, a substantial difference was found in the decay of the DR signal cross-correlation functions with growing frequency shift in the probing wave channels. The quick decrease in the radial correlation DR coherence observed in the experiment and full-wave synthetic diagnostic, compared to the fast synthetic DR, is attributed to the nonlinear effect of the probing wave phase modulation by the turbulence in the former two cases. The variation in the DR signal at a growing incidence angle in the experiment is also shown to be slower than predicted by both of the synthetic diagnostics, presumably due to underestimation of the probing wave phase modulation and consequent nonlinear saturation of the DR signal at lower incidence angles in modeling.
\end{abstract}

Keywords: gyrokinetic, Doppler reflectometry, synthetic diagnostic, radial correlation, phase modulation 


\section{Introduction}

Anomalous energy transport remains one of the main unresolved enigmas on the way to the realization of magnetic confinement fusion using the tokamak concept. According to the present day understanding $[1,2]$ anomalous transport is determined by multi-scale drift-wave turbulence and is strongly dependent on the nonlinear interaction of its components, such as large-scale mean $E \times B$ flows, meso-scale zonal flows and fine-scale micro-turbulence excited due to specific profiles of the plasma parameters. This interaction controlling anomalous transport has been the focus of theoretical and experimental research in magnetically confined plasmas for a long time. The massively parallelized particle-in-cell and semi-Lagrangian simulations of the gyrokinetic (GK) distribution function and the electric field [3,4] provide an efficient theoretical tool for studying nonlinear turbulent plasma dynamics, which, however, needs validation and comprehensive benchmarking against the experimental data. In recent papers $[5,6]$ such simulations of the electron and ion distribution function from the first principles were performed for the small research limiter tokamak FT-2. The complex interplay of the multi-scale turbulence components was studied in simulations, and successful quantitative comparison was presented with measurements carried out at the low magnetic field side of the machine with O-mode Doppler reflectometry (DR) and enhanced microwave scattering in the upper hybrid resonance $[5,6]$.

In the present paper the results of the global GK particlein-cell simulations will be quantitatively compared to the $\mathrm{X}$-mode DR experimental data obtained at the high magnetic field side of the FT-2 tokamak (these data characterize the tokamak turbulent dynamics and transport phenomena at different spatial and temporal scales). DR is the microwave diagnostic widely used nowadays in toroidal fusion experiments to obtain information on the multi-scale anomalous transport phenomena-namely, on the plasma rotation (both the mean value and its oscillations) and the turbulence poloidal wavenumber spectrum [7-11]. Recently, the radial correlation (RC) modification of this technique [12], providing information on the turbulence radial structure, was justified $[13,14]$.

Unfortunately, the interpretation of DR data is complicated due to the contribution of the poorly localized smallangle scattering along the wave trajectory, which can lead to overestimation of the turbulence RC length in the linear scattering regime $[13,14]$, and to its underestimation in the strongly nonlinear regime [15]. In the latter case the turbulence wavenumber spectrum measurements are questionable, and only the plasma turbulence mean velocity could be determined (however, with poor spatial resolution) [16]. Moreover, in the X-mode DR the turning point cannot be determined unambiguously due to $2 \mathrm{D}$ propagation effects, which additionally complicate the experimental data interpretation and their comparison with the theory predictions.

Development of a synthetic diagnostic that allows direct signal computation based on the results of GK modeling could be helpful in this case, both for interpretation of the experimental results and for the code benchmarking [17, 18]. In this paper two versions of the X-mode DR synthetic diagnostics are compared within the framework of the ELMFIRE global GK modeling of the FT-2 tokamak ohmic discharge [6]. The X-mode DR signal is computed both in the linear theory approximation using the reciprocity theorem, utilizing the probing wave field pattern provided by computation and taking into account the 2D plasma inhomogeneity effects [19], and by the full-wave code IPF-FD3D describing the probing and scattered wave propagation in turbulent plasma [20]. The temporal behavior of the DR signal frequency spectra and the dependence of its amplitude, frequency shift and shape on the probing antenna position are computed and compared to those measured in the experiment at the FT-2 tokamak. In the case of multi-frequency probing the RCDR cross-correlation function $(\mathrm{CCF})$ is also determined by both of the synthetic diagnostics and is compared to that obtained in the experiment.

\section{The experimental approach}

The experiment was performed at the FT-2 tokamak (the major radius $R=55 \mathrm{~cm}$, the limiter radius $a=7.9 \mathrm{~cm}$ ) in the hydrogen ohmic discharge (with plasma current $I_{\mathrm{p}}=19 \mathrm{kA}$, central density $n_{\mathrm{e}}(0)=4 \times 10^{13} \mathrm{~cm}^{-3}$ and electron temperature $T_{\mathrm{e}}=470 \mathrm{eV}$ ). The discharge is similar to that utilized for successful comprehensive benchmarking of the ELMFIRE GK code in $[5,6]$, however, the toroidal magnetic field at the discharge axis is slightly smaller $\left(B_{\mathrm{t}}(0)=1.7 \mathrm{~T}\right.$ instead of $2.1 \mathrm{~T})$. The actual measured electron density and temperature profiles for this discharge used in the GK modeling, as well as the ion temperature profile, are shown in figure 1 . The vertically movable (by $\pm 2 \mathrm{~cm}$ ) X-mode double antenna set (shown in figure 2), installed at the high magnetic field side in the vicinity of the equatorial plane, allowed plasma probing at a variable incidence angle with frequencies in the range $f_{\mathrm{i}}=(50-75) \mathrm{GHz}$. It was used both in standard DR measurements utilizing the quadrature scheme and in the RCDR technique based on the scheme described in [21]. In the former the DR spectrum was obtained by averaging 155 random DR power spectra obtained using Fourier transform of 12.8 microsecond samples of the DR signal. In the latter the reference channel generator was tuned to the master frequency $f_{0}=70$ $\mathrm{GHz}$ determining the measurement position in the vicinity of $r=5 \mathrm{~cm}$, whereas another generator (producing the probing signal at a slave frequency varied in the interval $\pm 1.2 \mathrm{GHz}$ around the master frequency by $100 \mathrm{MHz}$ steps) was used in the second (signal) channel to determine the turbulence twopoint CCF.

The backscattering (BS) spectra $P_{\mathrm{s}}(f)\left(f=f_{\mathrm{s}}-f_{\mathrm{i}}\right.$, where $f_{\mathrm{i}}$ and $f_{\mathrm{s}}$ are the probing and scattering frequencies, accordingly) measured by the X-mode DR diagnostics at different vertical antenna displacements were presented in [19]. It was shown there that the BS power decreases with the growing antenna vertical shift corresponding to the increase of the probing wave incidence angle, whereas the spectra frequency shifts and width increase. 


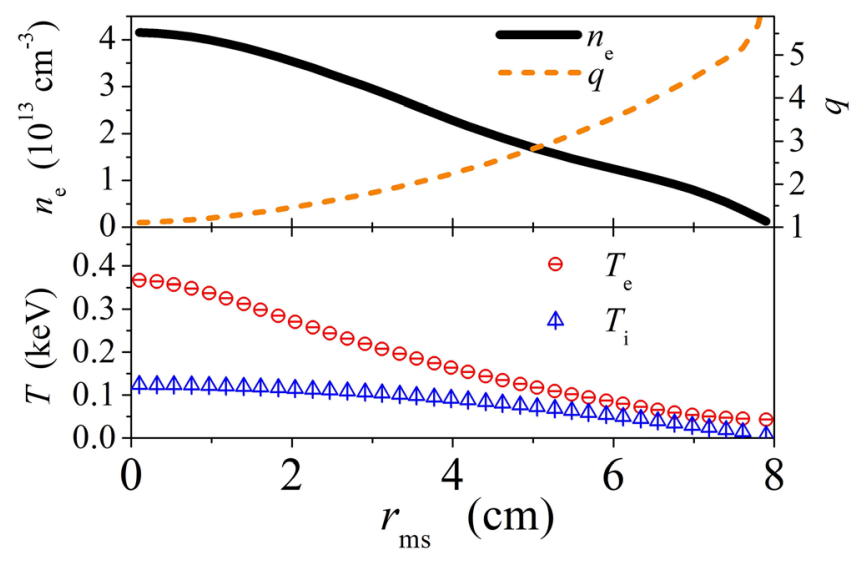

Figure 1. The discharge parameter profiles. Density—solid curve; safety factor-dashed curve. Electron temperature-circles; ion temperature-triangles.

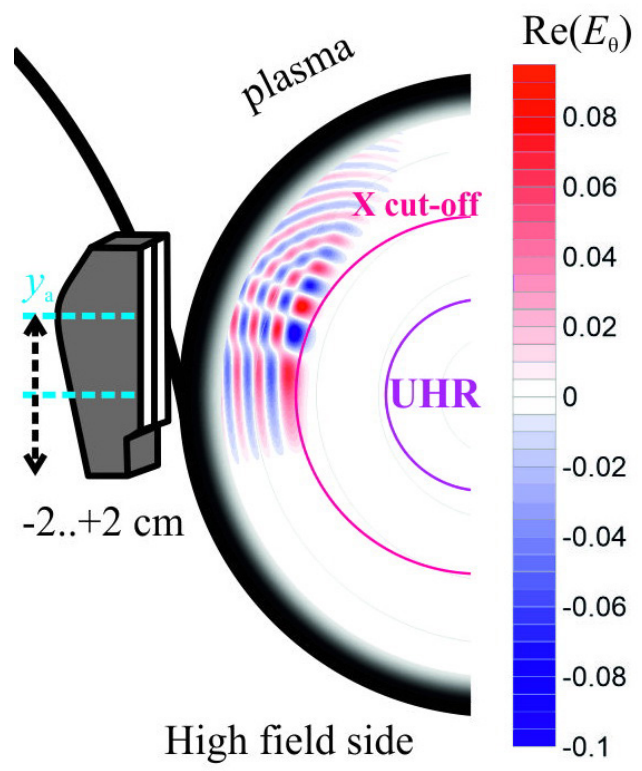

Figure 2. The DR experiment geometry and the probing wave poloidal electric field distribution computed at $y_{\mathrm{a}}=1.5 \mathrm{~cm}$.

\section{The GK computation approach and the synthetic diagnostics}

The simulations are performed with the global electrostatic particle-in-cell code ELMFIRE [3] to model the evolution of the full distribution function of drift kinetic electrons and GK protons and $\mathrm{O}^{6+}$ ions. The simulation geometry has a circular cross-section and covers the whole radial range. The time step is set to $\delta t=30 \mathrm{~ns}$ and the spatial grid to $110 \times 8$ cells in the radial $(r)$ and toroidal $(\varphi)$ directions, while the number of cells in the poloidal $(\theta)$ direction is set to increase with $r$ up to 950 at $r=7 \mathrm{~cm}$. The corresponding computational grid cell sizes at the minor radius $r=5 \mathrm{~cm}$ are as follows: $0.07 \mathrm{~cm}$ radial, and $0.07 \mathrm{~cm}$ poloidal. They are sufficient for an adequate description of fluctuations contributing to the DR signal in the experiment (the poloidal wavenumber is $0.4 \mathrm{~cm}^{-1}$ ) and correspond to the measured RC length (radial wavelength is $0.7 \mathrm{~cm}$ ). In the present version of the code [22] the parallel derivatives are calculated along the field lines (not as a combination of poloidal and toroidal derivatives at a given point). In practice this requires interpolation in the poloidal plane as the end points used in the derivative calculation are not grid nodes. As Hariri and Ottaviani [23] point out, with such a method, the coarse grid in the toroidal direction can be compensated by the fine grid in the poloidal direction carrying the information about the fine structure of the turbulence. The toroidal mode number is only the projection of parallel and poloidal components. However, modeling of smaller-scale turbulence, in particular, belonging to the electron temperature gradient (ETG) mode, is not possible with the ELMFIRE GK code. Approximately 2400 electrons and ions are simulated per grid cell. A momentum and energy conserving binary collision operator [24] is applied, allowing the inclusion of neoclassical physics in the simulations.

The particles are initialized according to a prescribed temperature and density profiles taken close to the experimentally measured ones (see figure 1). The profiles are allowed to develop self-consistently in time, as turbulence develops and heat sources and sinks, namely ohmic heating and radiation losses, are applied. The ohmic heating is induced by a spatially homogeneous loop voltage $[25,26]$, while electrons are cooled using a Monte Carlo model according to an impurity radiation loss distribution measured by the bolometry diagnostics. The initial oxygen $\mathrm{O}^{6+}$ impurity density is chosen to provide the experimental value of $Z_{\text {eff }}=2.2$, as determined using ASTRA modeling and experimental loop voltage measurements, with equal temperatures for the impurities and main ions. Neumann and Dirichlet boundary conditions are used at the boundary of the simulation domain for the electrostatic potential. Particles passing the boundary are returned to the simulation domain as an electron-ion pair according to a probability distribution proportional to the measured radial profile of neutral hydrogen density and at the wall temperature.

The fast synthetic X-mode DR diagnostic developed for this code [19] is based on the reciprocity theorem of electrodynamics [27], relating the high-frequency current in the plasma volume and the signal radiated by it and received by the antenna. The probing and BS wave propagation in the plasma not perturbed by turbulence is described in the fast synthetic diagnostic by the full-wave code WaveTOP2D [28]. The code solves complete Maxwell equations in the toroidal geometry accounting for the magnetic field shearing and presence of metallic walls, however, it suppresses reflections from the tokamak walls by specially introduced damping in the surface layer. The antenna beam is assumed Gaussian, as it was measured and computed at the air until the $-10 \mathrm{~dB}$ power level. Turbulent density fluctuations computed in the eight toroidal cross-sections were used to obtain the DR signal with the total duration $0.96 \mathrm{~ms}$. This realization was divided into $0.012 \mathrm{~ms}$ samples in which the random Fourier power spectra were calculated and then used to obtain the average DR power spectrum. It should be mentioned, however, that the approach based on the reciprocity theorem neglects the perturbations of the probing wave propagation caused by the density fluctuations; in particular, multiple small-angle scattering or its strong phase modulation. In this sense it is equivalent to the so-called Born approximation linear in the fluctuation amplitude. The main merit of this approach is the possibility of 


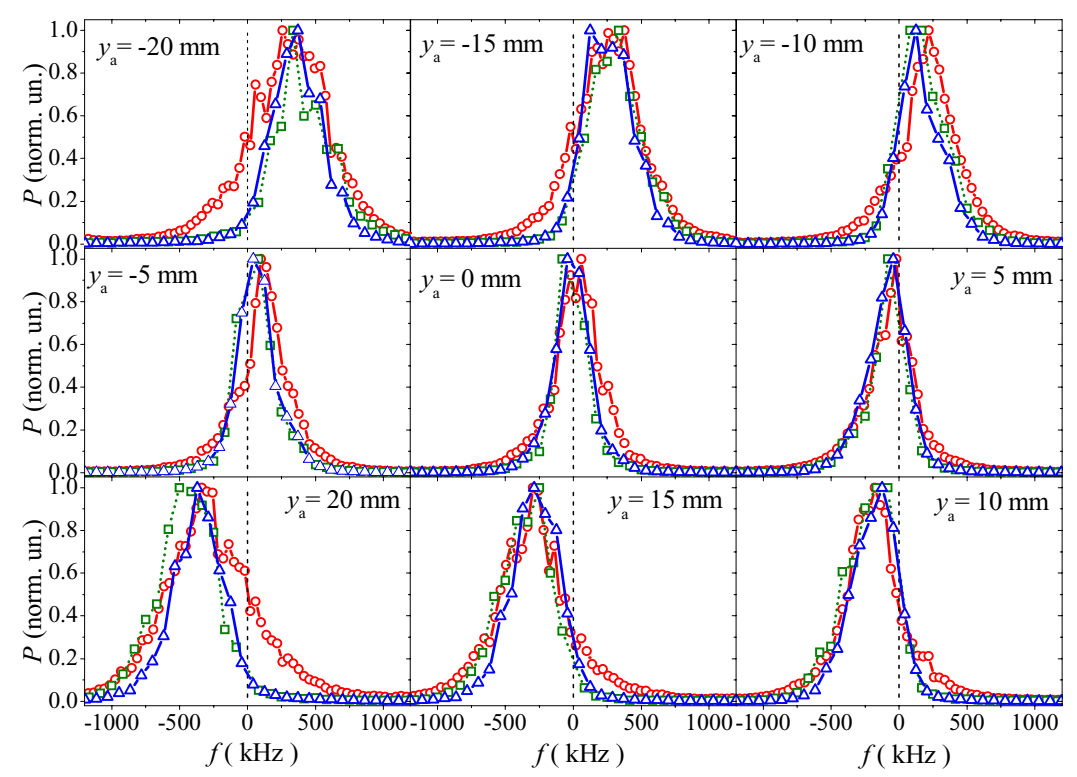

Figure 3. A comparison of DR spectra for different antenna vertical displacement. Circles—experiment; triangles-fast synthetic DR, squares-full-wave synthetic DR.

fast computations, whereas the main drawback is related to neglecting the nonlinear effects.

The alternative 'slow' DR synthetic diagnostic is free from this drawback. It is based on the full-wave code IPF-FD3D describing the probing and scattered wave propagation in turbulent plasma [20]. It accounts for the probing wave perturbations introduced by density fluctuations and, therefore, is not limited to the linear approximation. However, it solves the Maxwell equations with perfectly matched layer boundary conditions for a single polarization mode in the case of the propagation perpendicular to the magnetic field, the poloidal component of which is neglected as well as the toroidicity. It should be mentioned that multiple reflections between the plasma and the tokamak wall or microwave antenna, which could be important for the DR signal formation at small probing wave incidence angles, are not taken into account by either of the synthetic diagnostics.

It should be noted that the full-wave synthetic diagnostic simulations are very time demanding. With the full-wave code the computation of the synthetic reflectometry signal for a specific set of FT-2 tokamak experimental conditions requires around four hundred hours. This time scales with linear size as $(a / \lambda)^{3}$, where $a$ is the plasma size and $\lambda$ is the characteristic wavelength. The analogous computation by the fast synthetic diagnostic requires approximately four hours, but scaling with size is less favorable in this case- $(a / \lambda)^{4}$. In both cases computations are performed on a single Intel Xeon $2.4 \mathrm{GHz}$ CPU. Although full-wave synthetic diagnostics offer the possibility of parallelization and multi-frequency computation, they were not enough to overcome the drastic difference in computation time for the FT-2 experimental conditions.

\section{DR frequency spectra comparison}

The DR spectra obtained in the experiment and provided by the synthetic diagnostics are compared in figure 3 for vertical antenna shifts of different signs and values.
As shown in the figures, the spectra provided by both of the synthetic diagnostics fit reasonably well to the experimental ones for all the antenna positions. Only at the maximal antenna shifts do both of the synthetic diagnostics fail to describe the wing of the experimental spectrum opposite to the spectrum frequency shift direction. Nevertheless, not only are the shifts of both spectra close in all cases, but their shapes are also similar. This impression is confirmed by figures 4 and 5, where the mean frequency shift $f_{\mathrm{D}}=\int f \cdot P_{\mathrm{s}}(f) \mathrm{d} f / \int P_{\mathrm{s}}(f) \mathrm{d} f$ and the mean frequency width $\Delta f=\left[\int\left(f-f_{\mathrm{D}}\right)^{2} P_{\mathrm{s}}(f) \mathrm{d} f / \int P_{\mathrm{s}}(f) \mathrm{d} f\right]^{1 / 2}$ of the experimental and synthetic spectra obtained as the first and the second moment of the spectrum, respectively, are plotted as functions of the antenna vertical displacement and the turbulence poloidal wavenumber.

The relation between the antenna displacement and the fluctuation poloidal wavenumber was obtained using the X-mode ray tracing (as the doubled value of the probing wave poloidal wavenumber at the trajectory minimal radial coordinate). The spectral shift data provided by the experiment and GK modeling agree surprisingly well. A meaningful difference is only observed at the largest antenna displacement $y_{\mathrm{a}}= \pm 2.0 \mathrm{~cm}$. This difference is probably due to a contribution of experimental spectrum wings becoming especially heavy and asymmetric at high antenna displacement and, correspondingly, the fluctuation poloidal wavenumber. As was mentioned, the wing is opposite to the mean frequency shift of the spectrum and, presumably, is caused by small-scale drift modes excited in the plasma at a low level. These modes rotating in the direction of the ion diamagnetic drift might be poorly described by the GK code, since the smallest scales can be close to the limit of grid resolution and are also most sensitive to the particle noise. Furthermore, one should also note that in [21] for similar parameters we showed clear frequency mismatch, even with different signs for high poloidal wave numbers close to the edge when comparing the filtered ELMFIRE signal to the 


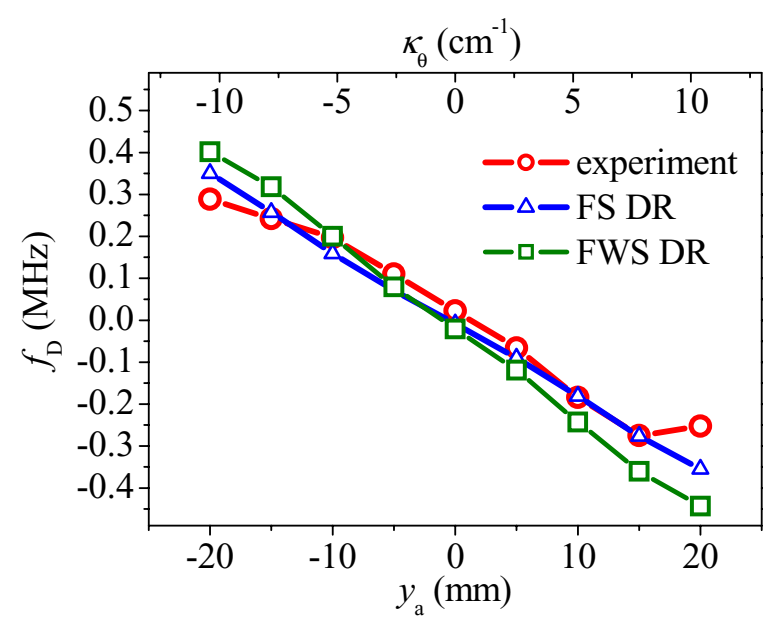

Figure 4. Dependence of the DR signal frequency shift on the antenna vertical displacement $y_{\mathrm{a}}$ and fluctuation wavenumber. Circles - experiment; triangles - fast synthetic DR; squares fullwave synthetic DR.

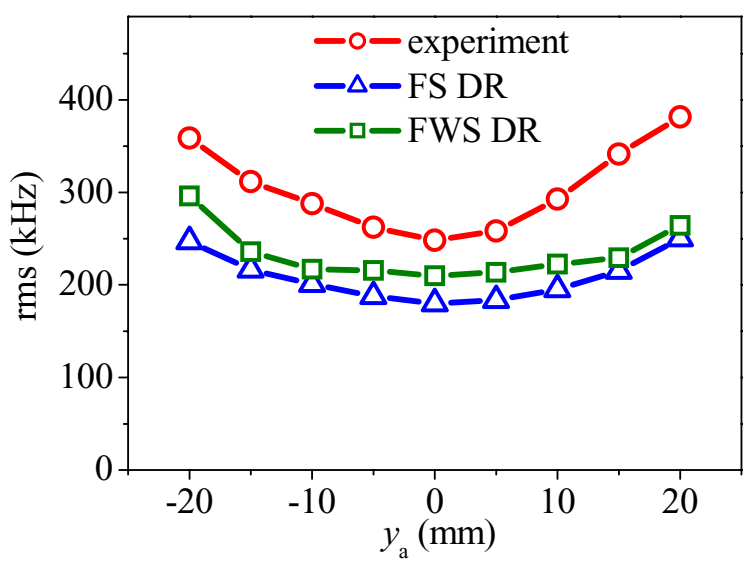

Figure 5. Dependence of the DR signal frequency width (rms) on the antenna vertical displacement $y_{\mathrm{a}}$. Circles-experiment; triangles-fast synthetic DR; squares full-wave synthetic DR.

linear GS2 analysis. Since the disagreement decreases when increasing the plasma current it was assumed to be caused by wide orbit effects, which were not included in the GS2 analysis. However, since the mode spectrum is very sensitive to the small changes in gradient lengths such disagreement can also be a result of the small changes in profiles during the full-f simulation. Another possible explanation of this difference could be associated with a non-Gaussian form of the experimental antenna beam at the $-10 \mathrm{~dB}$ level.

Figure 4 allows us to obtain estimations for the fluctuation poloidal velocity in the experiment and in the computation. The estimation of the mean fluctuation poloidal velocity in the experiment is given by $v_{\theta}=\pi f_{\mathrm{D}} / k_{\theta} \approx 2.1 \pm 0.2 \mathrm{~km} \mathrm{~s}^{-1}$, whereas the synthetic DR computations result in $v_{\theta} \approx 2.0 \pm 0.1 \mathrm{~km} \mathrm{~s}^{-1}$ for the fast diagnostics and $v_{\theta} \approx 2.4 \pm 0.1 \mathrm{~km} \mathrm{~s}^{-1}$ for the full-wave one. The obtained agreement between the measured and computed DR spectra, which are determined by the plasma flows, provides evidence in favor of correct modeling of plasma poloidal rotation and a correct reproduction of the radial electric field behavior in the FT-2 tokamak by the ELMFIRE code.

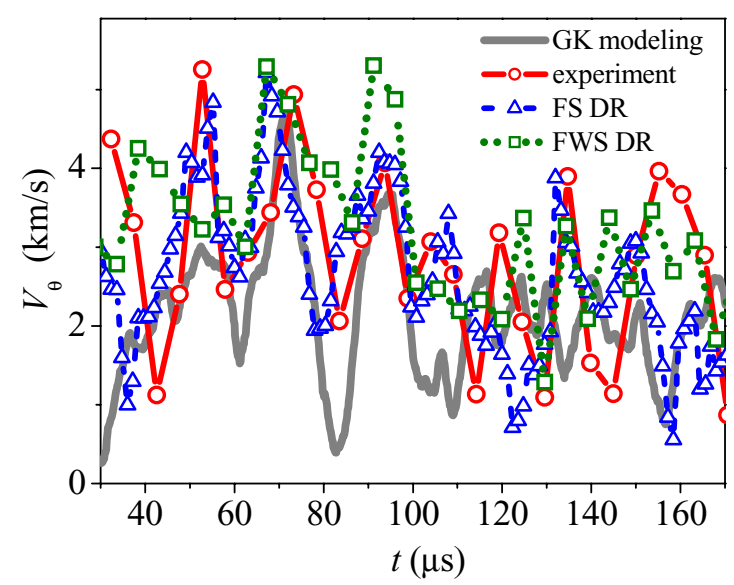

Figure 6. Poloidal velocity temporal behavior. Thick linecomputed directly by the GK code, circles - measured; trianglesprovided by fast synthetic diagnostics; squares-provided by fullwave synthetic diagnostics.

As shown in figure 5, the mean width of the synthetic spectra is systematically smaller than in the experiment. However, the difference is in the range of only 20\%-30\%. It could be attributed to the contribution of the spectral wings, which are higher in the experiment.

The large backscattering spectrum width observed in figure 5 is partly associated with strong plasma potential oscillations caused by the geodesic acoustic mode (GAM) excited in the FT-2 plasma [5, 6, 29]. These oscillations could be extracted from the DR signal using information on the temporal behavior of the frequency shift of its spectrum [7]. By utilizing $5 \mu$ s samples of the experimental and synthetic DR signal the following dynamics of the plasma poloidal velocity shown in figure 6 were obtained. The oscillations in the $v_{\theta}(t)$ behavior are caused by the GAM. The velocity oscillation amplitudes provided by the experimental measurement, fast and full-wave synthetic diagnostics are close $\left(1.7 \pm 0.3 \mathrm{~km} \mathrm{~s}^{-1}\right.$; $1.6 \pm 0.3 \mathrm{~km} \mathrm{~s}^{-1}$ and $1.4 \pm 0.2 \mathrm{~km} \mathrm{~s}^{-1}$, respectively). The amplitude of the plasma poloidal velocity oscillations determined directly from the GK code output using the magnetic surface averaged radial electric field is also close to these values $\left(1.3 \pm 0.5 \mathrm{~km} \mathrm{~s}^{-1}\right)$. The standard deviations of the velocity fluctuations determined using DR data are also similar: the values $1.1 \mathrm{~km} \mathrm{~s}^{-1}, 0.9 \mathrm{~km} \mathrm{~s}^{-1}, 1.0 \mathrm{~km} \mathrm{~s}^{-1}$ and $0.8 \mathrm{~km} \mathrm{~s}^{-1}$ were obtained in the experiment, given by the two synthetic diagnostics and directly provided by the GK code. The GAM is represented by a line in the Fourier spectrum of velocity shown in figure 7. As shown in the figure, the experimental value of the GAM frequency is $40 \mathrm{kHz}$, and the computational value provided by the fast synthetic diagnostic is slightly higher at $45 \mathrm{kHz}$; whereas the value given by the full-wave synthetic diagnostic is lower- $36 \mathrm{kHz}$. The GAM frequency given directly by the code $(42 \mathrm{kHz})$ is also close to the experimental value.

We have also compared the DR signal intensity $\int P_{\mathrm{s}}(f) \mathrm{d} f$ dependence on the vertical antenna displacement, which is expressed in figure 8 in terms of the poloidal wavenumber of the turbulence. As shown in figure 8, the decrease in the DR signal power with growing fluctuation poloidal wavenumber 


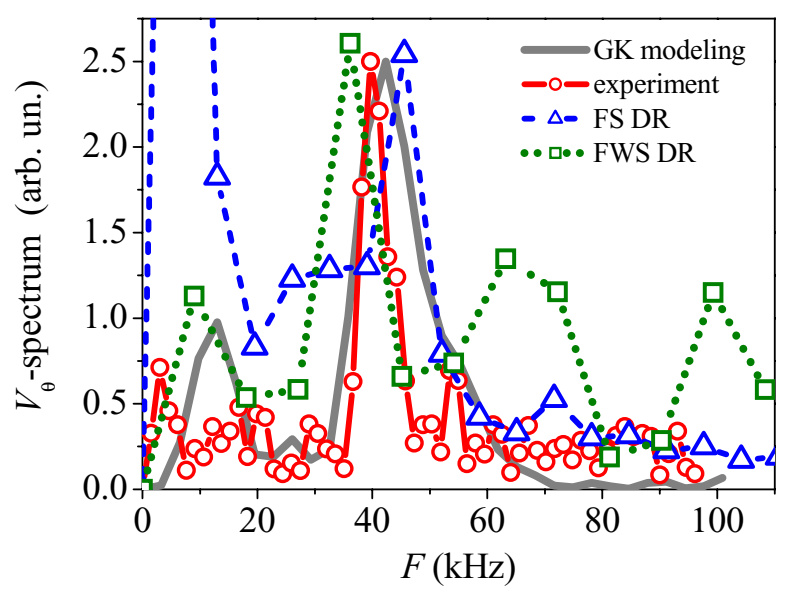

Figure 7. Power frequency spectra of the velocity oscillations shown in figure 6 . Thick line-computed by the GK code, circlesmeasured; triangles - provided by fast synthetic diagnostics; squares-provided by full-wave synthetic diagnostics.

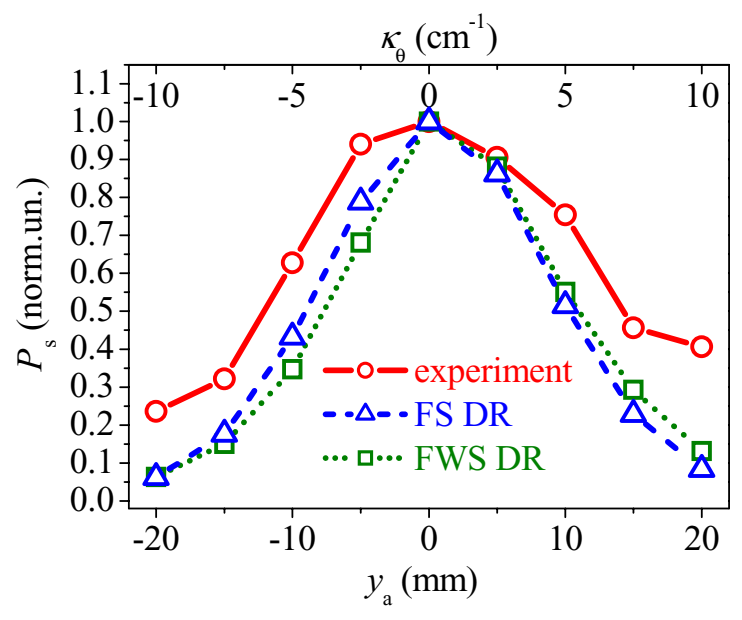

Figure 8. Dependence of the BS power on the fluctuation poloidal wavenumber. Circles - experiment; triangles—fast synthetic DR; squares-full-wave synthetic DR.

is substantially faster than in the experiment for both synthetic diagnostics. The difference could be attributed to at least two possible reasons. The first reason is related to incorrect modeling of the ETG mode-scale fluctuations leading to their suppression, and absence of the inverse energy cascade in the wavenumber space, which can also result in an underestimation of the turbulence poloidal wavenumber spectrum in the intermediate-scale domain $\kappa_{\vartheta} \simeq 10 \mathrm{~cm}^{-1}$. It can occur in spite of sufficiently high density of the GK code grid (poloidal cell size $0.07 \mathrm{~cm}$ ). This could lead to the faster decay of the synthetic signal power at large antenna displacement. The second reason is related to a possible underestimation by the GK code of the total density fluctuation level. As we show in the next section, this could reduce the role of nonlinear effects: in particular, it could slow down the saturation of the computed DR signal growth with the turbulence level increase at small antenna displacements. The same consequences could result from neglecting in full wave synthetic diagnostic multiple wave reflections between the cutoff and the antenna taking place at the equatorial probing.

\section{RCDR CCF comparison}

Inspired by a reasonable agreement of the experimental and synthetic DR spectral data we have performed a comparison of the computed and measured RCDR CCFs using the X-mode DR antenna displaced by $+2.0 \mathrm{~cm}$ from the equatorial plane at the high-field side of the FT-2 tokamak. The corresponding CCFs (shown in figure 9 as functions of the frequency difference in the master and slave channels $(a)$ and of the separation of turning points $(b)$, which was determined using ray tracing for different probing frequencies, as the difference of minimal radii on the two trajectories) appear to be different, whereas the density fluctuations two-point $\mathrm{CCF}$ for frequency $f_{\mathrm{s}}-f_{\mathrm{i}}=300 \mathrm{kHz}$ computed in the measurement region based on the GK data and shown in figure $9(b)$ is close to the measured RCDR CCF, as was mentioned in [21]. However, this coincidence is just accidental. The CCF provided by the fast synthetic DR is much wider than the experimental one, whereas the full-wave synthetic CCF is situated in between, as shown in figure $9(a)$. The slow decay of the CCF provided by fast synthetic diagnostics shown in figures $9(a)$ and $(b)$ is most likely determined by the small-angle-scattering contribution to the DR signal [13], which is not suppressed at the angle of incidence corresponding to $y_{\mathrm{a}}=+2.0 \mathrm{~cm}$. There are two possible reasons for the drastic difference between the experimental and the fast synthetic RCDR CCFs. The first one is related to the influence of nonlinear effects; in particular, the multiple small-angle scattering, or strong probing wave phase modulation, according to theory $[15,16,30]$, coming into play with the growing turbulence level. The importance of this effect for the DR diagnostics' performance was shown recently at the ASDEX Upgrade [9]. The second reason is related to the GK code spatial grid limitations leading to an underestimation of the turbulence radial wavenumber spectrum width, and thus, to the overestimation of the RCDR CCF width.

The role of the probing wave phase modulation in the FT-2 $\mathrm{X}$-mode RCDR experiment was studied in [31], where the random phase perturbation of the backscattered wave was computed using the geometrical optics approximation and measured. The standard deviation of the computed DR signal phase was shown to be 0.93 , whereas for the measured phase it is equal to 1.23 . The latter value is close to the computed one and to the level $\pi / 3$, which indicates the borderline for the DR diagnostics' transition into the nonlinear regime of operation. Strictly speaking, in this parameter domain the reciprocity theorem is not applicable. The obtained agreement of the measured and computed DR signal random phase standard deviation and its relatively high value demonstrates the importance of the first, based upon nonlinear effects, explanation of the drastic disagreement of the experimental and fast synthetic RCDR diagnostics results, and appeals for the application of the full-wave synthetic diagnostics.

As shown in figure $9(a)$, the CCF provided by the fullwave synthetic diagnostics is much closer to the experimental one. This agreement shows that the second reason mentioned above probably plays a minor role, thus providing an argument in favor of a correct description of the turbulence radial wavenumber spectrum by the GK code with the utilized grid. 

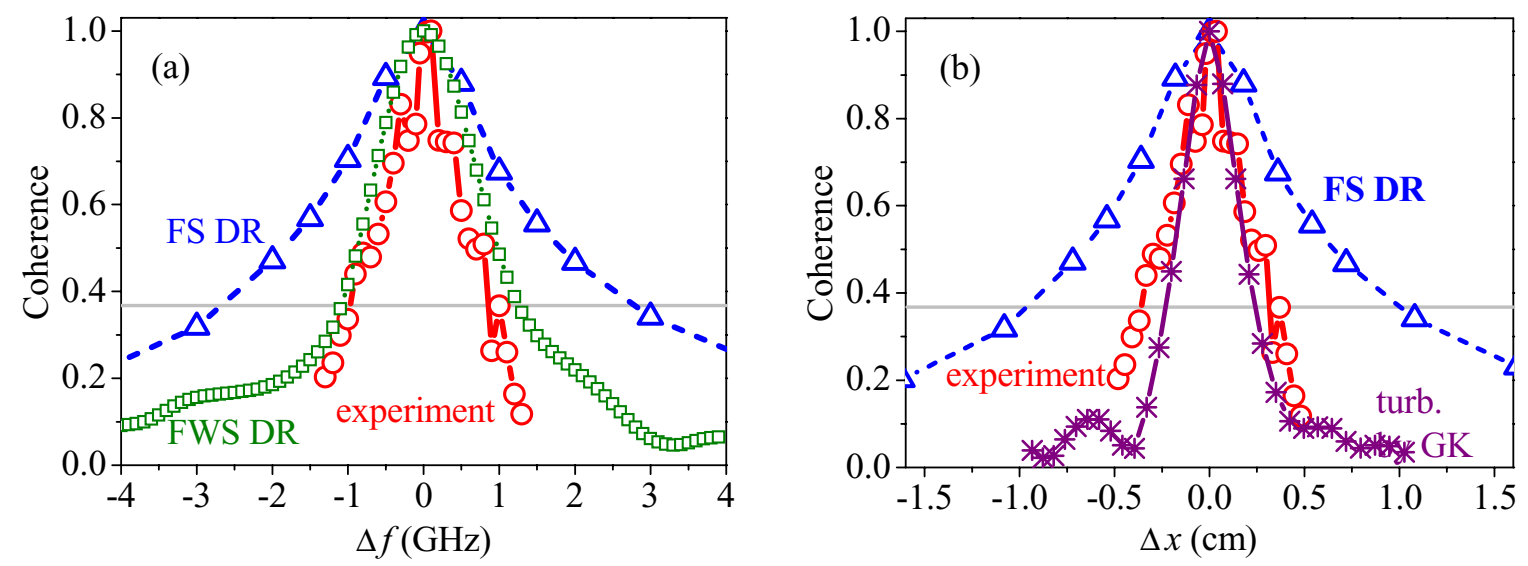

Figure 9. (a) The RCDR CCF against the channel frequency separation. Triangles-fast synthetic DR; squares-full-wave synthetic DR; circles-experiment. (b) The RCDR CCF against the turning point radial separation. Triangles—fast synthetic DR; circles—experiment; double crosses - turbulence CCF by the GK code.

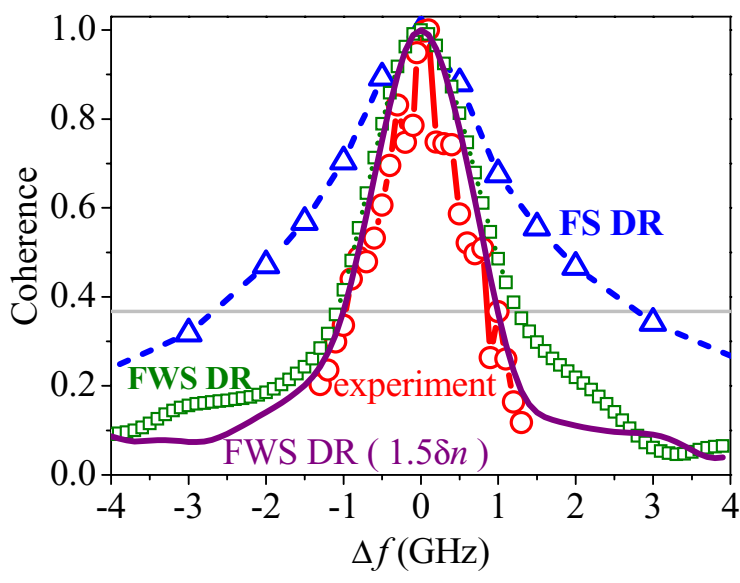

Figure 10. The RCDR CCF against the channel frequency separation. Triangles - fast synthetic DR; squares-full-wave synthetic DR; circles—experiment; solid line-FWS DR with the density fluctuation level multiplied by 1.5 .

It should be mentioned that an artificially imposed increase in the density fluctuation level provided by the GK code by a factor of 1.5 , roughly corresponding to the excess of the experimental DR signal phase root mean square over the computed one, results in an even better agreement of the experimental and synthetic CCF shown in figure 10. At such a high level of fluctuations the DR already operates in the saturation regime for antenna positions close to the equatorial plane, which leads to the DR signal power growth with the fluctuation level weaker than the quadratic. The same consequences could result from the aforementioned multiple wave reflections neglected in both synthetic diagnostics. The dependence of the DR signal power on the antenna vertical shift in this case is shown in figure 11. Due to the saturation of the DR signal growth close to the equatorial plane the synthetic dependence provided by the full-wave diagnostics in this case appears to be much closer to the experimental one, thus confirming a qualitative explanation for the disagreement given in the previous section. It should also be noted that the increase in the turbulence level by a factor of 1.5 did not alter the DR signal frequency spectra, which remained similar to the experimental spectra. Thus, the threshold of the strong influence of the strong

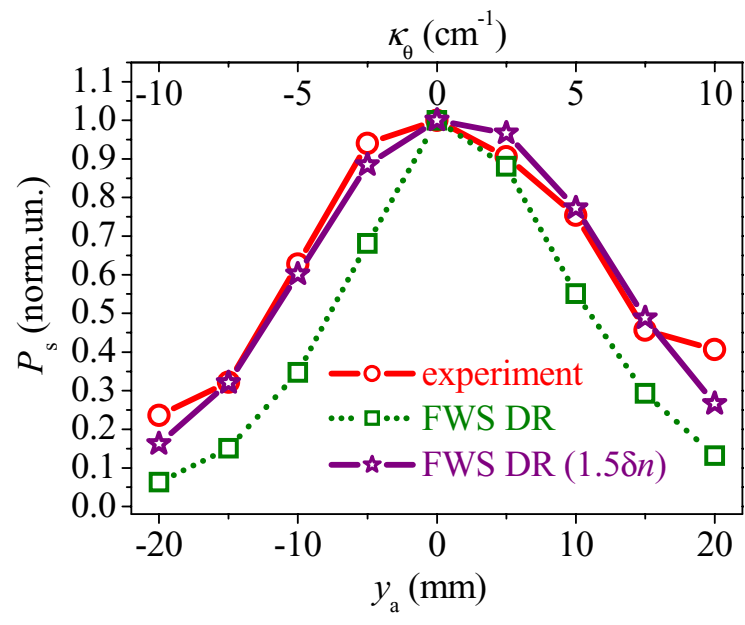

Figure 11. Dependence of the BS power on the fluctuation poloidal wavenumber. Circles - experiment; squares-full-wave synthetic DR; stars-FWS DR with the density fluctuation level multiplied by 1.5 .

probing wave phase modulation on the width of the Doppler frequency spectra in the FT-2 case substantially exceeds the corresponding thresholds for the RCDR and poloidal wavenumber spectra measurements. This result appears to be in agreement with theoretical expectations [15, 30], because the latter thresholds are determined just by transition of reflectometry to the saturation regime, whereas for the former the spectral broadening of the probing wave due to strong phase modulation obtained in [30] should exceed broadening due to other mechanisms. Namely, in the FT-2 experiment it should exceed the broadening due to the strong GAM excitation level, which is dominant.

\section{Conclusions}

Based on the results of the paper one should conclude that the ELMFIRE code benchmarking against the FT-2 X-mode DR experimental data, utilizing the fast linear and full-wave DR synthetic diagnostics, has demonstrated good agreement between the measured and computed DR frequency spectra. 
For all used incidence angles both the spectra frequency shift and width, and in many cases the spectra shapes, were similar. A meaningful difference was only observed at the largest antenna displacement. This difference is probably due to a contribution of small-scale drift modes excited in the plasma at a low level. These modes rotating in the direction of the ion diamagnetic drift are probably not described well by the GK code. Another possible explanation for this difference could be associated with a non-Gaussian form of the antenna beam at the $-10 \mathrm{~dB}$ level.

The mean fluctuation velocities in the DR measurements and in the synthetic diagnostics were close. The GAM characteristics provided by the measurements and by the synthetic DR also appear to be close.

However, the variation in the DR signal power with growing incidence angles in the experiment is slower than that predicted by both of the synthetic diagnostics. This difference could be presumably attributed to the underestimation of the turbulence level and related DR signal saturation effect, and to the overestimation of the turbulence poloidal wavenumber spectrum decay rate with growing wavenumber due to an incorrect description of the ETG mode-scale domain in the GK modeling. The same effect could result from multiple wave reflections between the cutoff and the antenna taking place at the equatorial probing being neglected by the full-wave synthetic diagnostics, as well as by the fast one.

The substantial difference was also found in the decay of the DR signal CCFs with growing frequency shift in the probing wave channels. The quick decrease in the RCDR coherence observed in the experiment and full-wave synthetic diagnostic compared to the fast synthetic RCDR is attributed to nonlinear effects, which manifest themselves in the phase modulation of the probing wave in the former two cases. It was shown to be close to $\pi / 3$, both in the specially performed measurements and in the GK modeling [31]. In spite of the fact that this value indicates only the beginning of the transition to the fluctuation reflectometry nonlinear regime (and therefore has no impact on the DR spectra) it already has a strong influence on the RCDR performance.

\section{Acknowledgments}

The financial s upport of the Russian $\mathrm{S}$ cience Foundation grant 17-12-01110 is acknowledged. The work has been supported by the Academy of Finland grants 278487, 296853 and 318314. CSC-IT Center for Science and Marconi-Fusion HPC are acknowledged for generous allocation of computational resources for this work. The FT-2 tokamak maintenance was supported by the Ioffe Institute, whereas M.A. Irzak acknowledges the support of the Russian Academy of Science Presidium program

\section{ORCID iDs}

O.L. Krutkin (1) https://orcid.org/0000-0002-9124-2659

A.B. Altukhov (1) https://orcid.org/0000-0002-5913-6004

A.D. Gurchenko (1) https://orcid.org/0000-0002-2540-2511

T.P. Kiviniemi (1) https://orcid.org/0000-0001-9753-3388

P. Niskala (1) https://orcid.org/0000-0001-7308-9921

C. Lechte (1) https://orcid.org/0000-0003-4171-888X

\section{References}

[1] Diamond P.H. et al 2005 Plasma Phys. Control. Fusion 47 R35

[2] Terry P.W. 2000 Rev. Modern Phys. 72109

[3] Heikkinen J.A. et al 2008 J. Comput. Phys. 2275582

[4] Sarazin Y. et al 2011 Nucl. Fusion 51103023

[5] Leerink S. et al 2012 Phys. Rev. Lett. 109165001

[6] Gusakov E.Z. et al 2013 Plasma Phys. Control. Fusion 55124034

[7] Conway A.D. et al 2005 Plasma Phys. Control. Fusion 471165

[8] Hennequin P. et al 2004 Plasma Phys. Control. Fusion 46 B 121

[9] Happel T. et al 2017 Plasma Phys. Control. Fusion 59054009

[10] Hillesheim J.C. et al 2012 Phys. Plasmas 19022301

[11] Vermare L. et al 2018 Phys. Plasmas 25020704

[12] Schirmer J. et al 2007 Plasma Phys. Control. Fusion 491019

[13] Gusakov E., Irzak M. and Popov A. 2014 Plasma Phys. Control. Fusion 56025009

[14] Gusakov E. et al 2017 Phys. Plasmas 24022119

[15] Gusakov E.Z. and Popov A.Yu. 2004 Plasma Phys. Control. Fusion 461393

[16] Gusakov E.Z., Surkov A.V. and Popov A.Yu. 2005 Plasma Phys. Control. Fusion 47959

[17] Hacquin S. et al 2016 Phys. Plasmas 23092303

[18] Citrin J. et al 2017 Phys. Plasmas Control. Fusion 59064010

[19] Altukhov A.B. et al 2018 Phys. Plasmas 25082305

[20] Lechte C. et al 2017 Plasma Phys Control. Fusion 5907500

[21] Altukhov A.B. et al 2016 Plasma Phys. Control. Fusion 58105004

[22] Korpilo T. et al 2016 Comput. Phys. Commun. 203 128-37

[23] Hariri F. and Ottaviani M. 2013 Comput. Phys. Commun. 1842419

[24] Takizuka T. et al 1977 J. Comput. Phys. 25205

[25] Kiviniemi T.P. et al 2014 Plasma Phys. Control. Fusion 56075009

[26] Leerink S. 2012 PhD Thesis Aalto University (http://lib.tkk.fi/ Diss)

[27] Piliya A.D. and Popov A.Yu. 2002 Plasma Phys. Control. Fusion 44467

[28] Gusakov E.Z. et al 2010 Plasma Phys. Control. Fusion 52075018

[29] Gurchenko A.D. et al 2013 Plasma Phys. Control. Fusion 55085017

[30] Gusakov E.Z. and Popov A.Yu. 2002 Plasma Phys. Control. Fusion 442327

[31] Altukhov A.B. et al 2018 Phys. Plasmas 25112503 